\title{
Attenuation Correction in Myocardial Perfusion SPECT/CT: Effects of Misregistration and Value of Reregistration
}

\author{
Sibyll Goetze ${ }^{1,2}$, Tracy L. Brown ${ }^{1}$, William C. Lavely ${ }^{1}$, Zhe Zhang ${ }^{3}$, and Frank M. Bengel ${ }^{1}$ \\ ${ }^{I}$ Division of Nuclear Medicine, Johns Hopkins University, Baltimore, Maryland; ${ }^{2}$ Division of Nuclear Medicine, University of Alabama \\ at Birmingham, Birmingham, Alabama; and ${ }^{3}$ Division of Biostatistics, Johns Hopkins University, Baltimore, Maryland
}

The accuracy of myocardial perfusion SPECT improves with attenuation correction. Algorithms for attenuation correction in hybrid SPECT/CT systems have the potential for misregistration of emission and transmission scans because CT and SPECT are obtained sequentially. Misregistration will influence regional tracer distribution and may reduce diagnostic accuracy. This study focused on the role of misregistration in cardiac SPECT/ CT and the performance of a software-based approach for reregistration. Methods: We included 105 consecutive patients who underwent clinical myocardial perfusion imaging on a SPECT/ CT system. Images were quantitatively assessed for misregistration using fusion software. Results were recorded in millimeters in the $x-, y$-, and $z$-axes. Regional tracer uptake in 6 segments (anterior, septal, inferior, lateral, anteroapical, and inferoapical) for noncorrected and attenuation-corrected images before and after reregistration was obtained from polar maps. To determine the relative influence of misregistration, we correlated individual differences between noncorrected and attenuation-corrected images, as well as between attenuation-corrected images before and after reregistration, with the degree of misregistration in a multivariate analysis including additional clinical variables such as sex and body weight. Results: The difference in regional radiotracer uptake was significant between noncorrected and attenuationcorrected images in all 6 segments and was most pronounced in the inferior wall. On multivariate analysis, misregistration contributed significantly to changes in radiotracer distribution in the anterior $(P=0.038)$, septal $(P=0.011)$, and inferior $(P=0.006)$ segments. The mean misregistration was $8.6 \pm 3.8 \mathrm{~mm}(1.25 \pm$ 0.55 pixel). Misregistration of one or more pixels was observed in $64 \%$ of studies. Reregistration of misalignment significantly affected regional radiotracer distribution in the segments shown to be influenced by misregistration. Conclusion: Misregistration occurs with SPECT/CT systems and influences regional tracer distribution on attenuation-corrected myocardial images. Reregistration of misaligned studies may be a useful tool for correction. The impact of this strategy on the diagnostic and prognostic accuracy of cardiac hybrid imaging needs to be determined.

Received Feb. 5, 2007; revision accepted Mar. 19, 2007.

For correspondence contact: Frank M. Bengel, MD, Division of Nuclear Medicine/PET, 601 N. Caroline St., JHOC 3225, Baltimore, MD 21287-0817. E-mail: fbengel1@jhmi.edu

COPYRIGHT @ 2007 by the Society of Nuclear Medicine, Inc.
Key Words: SPECT/CT; hybrid imaging; attenuation correction; myocardial perfusion imaging

J Nucl Med 2007; 48:1090-1095

DOI: 10.2967/jnumed.107.040535

$\mathbf{F}$ or myocardial perfusion SPECT, professional societies recommend incorporation of attenuation correction to improve diagnostic accuracy (1). On standalone SPECT scanners, the transmission scans necessary for determining tissue density maps for attenuation correction are obtained with an external radionuclide line or point source. With the advent of SPECT/CT hybrid systems, however, CT is now increasingly used for transmission scanning. Advantages of the CT method include higherquality attenuation maps secondary to higher photon flux, lower noise, and improved resolution (2). Additionally, especially when using multislice CT in SPECT/CT systems, coronary calcium scoring or coronary angiography can be performed together with myocardial perfusion assessment in the same session.

A disadvantage of the use of CT for attenuation correction in SPECT/CT is the potential for misregistration of emission and transmission scans because CT and SPECT are obtained sequentially. Possible sources of misregistration include improper patient positioning, respiratory motion, other voluntary patient movement, or mechanical misalignment of the SPECT/CT device. Phantom and human studies have shown that misregistration can produce errors in the activity distribution of attenuation-corrected perfusion images (3-6). This change in regional radiotracer distribution may reduce the diagnostic accuracy of myocardial perfusion SPECT.

To address this issue, software tools for reregistration based on visual alignment of SPECT/CT fusion images have been developed. The objective of this study was to quantitatively determine the role of misregistration and the performance of reregistration in cardiac SPECT/CT.

\section{MATERIALS AND METHODS}

\section{Patients}

From a total of 124 consecutive patients who underwent myocardial perfusion scanning at our institution over a 1-mo period, 
we included 105 patients who underwent CT attenuation-corrected myocardial perfusion studies at rest or stress. Nineteen patients were excluded: Nine refused CT because of claustrophobia, and the remaining 10 had CT studies with significant artifacts-motion, metal, or beam-hardening-precluding evaluation of registration or of attenuation-corrected myocardial perfusion images.

Retrospective analysis of the imaging data was granted exempt status by the Johns Hopkins institutional review board, and informed consent was waived.

\section{SPECT/CT Protocol}

Myocardial perfusion imaging was performed according to standard clinical protocols for a 1-d rest-stress study with a single tracer $\left({ }^{99 \mathrm{~m}} \mathrm{Tc}\right.$-sestamibi; $296 \mathrm{MBq}$ at rest and $925 \mathrm{MBq}$ to 1.48 $\mathrm{GBq}$ at stress) or 2 tracers $\left({ }^{201} \mathrm{Tl}, 111 \mathrm{MBq}\right.$ at rest, and ${ }^{99 \mathrm{~m}} \mathrm{Tc}-$ sestamibi, $925 \mathrm{MBq}$ to $1.48 \mathrm{GBq}$ at stress). All studies were acquired on 2 Millennium VG/Hawkeye SPECT/CT systems (GE Healthcare), referred to as camera 1 and camera 2 in the "Results" section. The scans were obtained sequentially, with CT obtained immediately after each SPECT study on the hybrid system.

SPECT was done using a low-energy, high-resolution, parallelhole collimator and the following specifications: $90^{\circ}$ per head from the right anterior oblique to left anterior oblique positions; 60 stops (step-and-shoot mode); 25 s per stop; and $64 \times 64$ matrix reconstruction. For the attenuation map, single-slice nonspiral CT (x-ray tube current, $2.5 \mathrm{~mA}$; voltage, $140 \mathrm{kVp}$ ) with a slice thickness (and axial resolution) of $10 \mathrm{~mm}$ and a scan time of more than $5 \mathrm{~min}$ for a typical $13-\mathrm{cm}$ field of view was obtained without intravenous contrast material and without a breath-hold.

\section{Quantification of Misregistration and Reregistration}

Images were retrospectively and independently reviewed by 3 readers ( 1 experienced, 1 less experienced, and 1 novice), using a dedicated image analysis workstation (Xeleris; GE Healthcare). Datasets were quantitatively assessed for misregistration between SPECT and CT using a fusion/registration software tool provided by the manufacturer (ACQC tool; GE Healthcare), which allows manual shifting of SPECT versus CT in 3 dimensions. Reregistra- tion was performed by moving the CT scan in the $x$-, $y$-, and $z$-axes relative to the SPECT scan, to match the borders of the left ventricle on both studies (Fig. 1). Necessary shifts were displayed in millimeters and recorded for further analysis. The amount of misregistration in a dataset was defined as the maximum misalignment observed in any dimension of space based on the results of the most experienced reader. In addition to the maximal misalignment, the Euclidian distance of misalignment in all 3 dimensions of space $\left(\sqrt{ }\left(x^{2}+y^{2}+z^{2}\right)\right)$ was calculated.

\section{Regional Myocardial Radiotracer Uptake}

From reangulated tomographic images, polar maps of regional myocardial tracer uptake (in percentage of maximal left ventricular pixel intensity) were generated using commercially available software (Quantitative Perfusion SPECT; Cedars-Sinai). From noncorrected images, and attenuation-corrected images before and after reregistration, average tracer uptake was measured in 6 polar map segments (anterior, septal, inferior, lateral, anteroapical, and inferoapical).

\section{Statistical Analysis}

Results are described as mean \pm SD. A $P$ value of less than 0.05 was considered statistically significant. $\kappa$-statistics were obtained to define inter- and intraobserver variability. Differences between groups were assessed by an unpaired Student $t$ test. Linear regression analysis was performed to describe the relationship between continuous variables. To determine whether the individual degree of misalignment was an independent determinant of regional perfusion tracer uptake on attenuation-corrected images, we performed a multivariate analysis. A mixed-effects model was used for each of the 6 myocardial segments to analyze the influence on changes in radiotracer uptake between noncorrected and nonrealigned attenuation-corrected images, as well as between attenuation-corrected images before and after realignment. Besides the degree of misregistration, the clinical covariates that were entered into the analysis were the sex, age, and weight of the patients; rest or stress status; radiotracer used; and camera used.

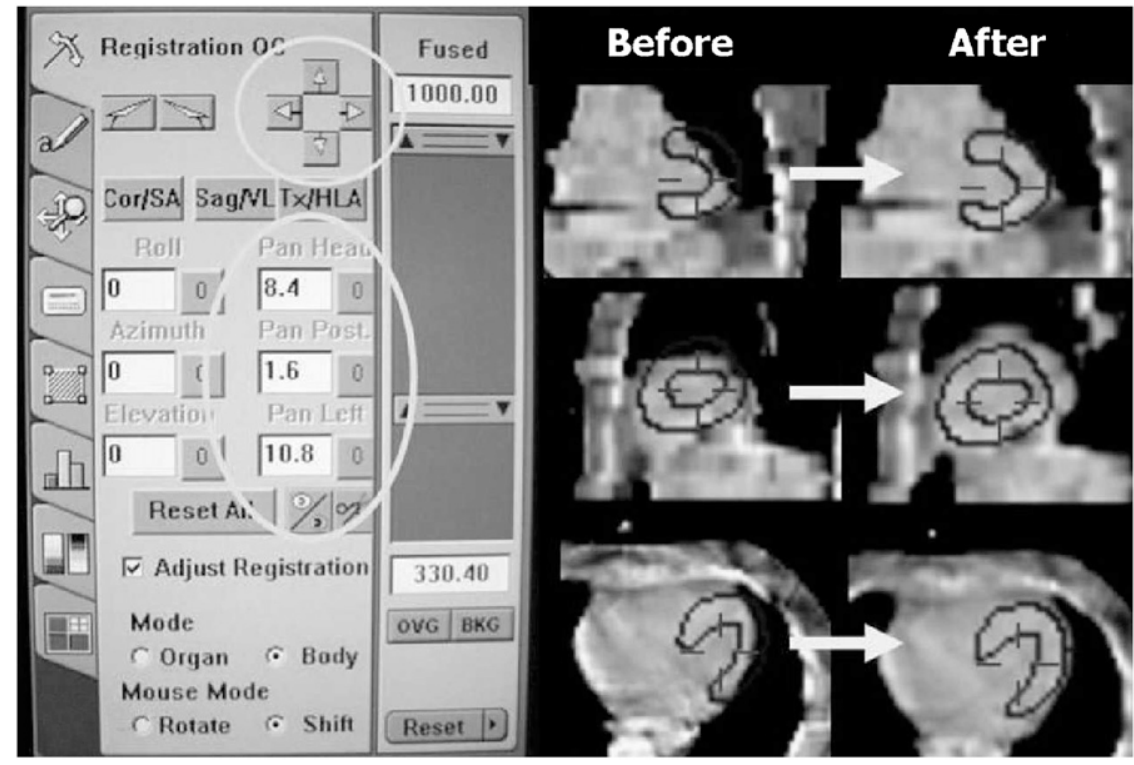

FIGURE 1. Software tool for realignment of misregistered CT and SPECT data. Using arrows of toolbar (left), CT can be moved in $x$-, $y$-, and $z$-axes relative to SPECT in order to match borders of left ventricle. In fusion images on right, dark lines depict myocardial borders of SPECT scan. In present case, CT was moved left and cranially to obtain alignment as illustrated by fused images in coronal, sagittal, and transaxial planes before and after realignment. Shifts are displayed in millimeters on toolbar and recorded for further analysis. 


\section{RESULTS}

The 105 patients included 51 female and 54 male patients (mean age, $56 \pm 13.1 \mathrm{y}$; range, 33-87 y). The average weight was $85.5 \pm 16.3 \mathrm{~kg}$ (range, $50-145.9 \mathrm{~kg}$ ). Most patients $(91 / 105,87 \%)$ had both arms above the head for SPECT/CT, $10 \%(11 / 105)$ had the right arm down or the left arm above the head, and 3\% (3/105) had both arms at the sides. CT for attenuation correction was available for rest and stress studies in 94 patients, whereas 11 patients had only rest or stress attenuation-corrected images, resulting in a total of 199 image datasets. These included 100 stress and 99 rest datasets, 74 scans with ${ }^{201} \mathrm{Tl}$, and 125 scans with ${ }^{99 \mathrm{~m}} \mathrm{Tc}$-sestamibi. Ninety-seven scans were performed on camera 1 and 102 on camera 2 .

\section{Misregistration}

The mean recorded maximal misregistration was $8.6 \pm$ $3.8 \mathrm{~mm}$, with a range of $0-22.4 \mathrm{~mm}$. This correlated significantly with the Euclidian distance of misalignment, which was $10.2 \pm 4.3 \mathrm{~mm}$, with a range of $0-25.1 \mathrm{~mm}(r=$ $0.97 ; P<0.001)$. Hence, the maximal misregistration was used for further analysis, based on the assumption that regional tracer distribution is most influenced by the maximal misalignment in a single direction of space.

With a known pixel size of $6.9 \mathrm{~mm}$ for the SPECT system, maximal misregistration equaled $1.25 \pm 0.55$ pixels. Seventy-one studies (36\%) showed less than 1 pixel of misalignment, 113 studies (57\%) showed a misalignment of 1-2 pixels, and 15 studies (7\%) showed a misalignment of at least 2 pixels. Most frequently, the CT scan had to be shifted along the $x$-axis to the left $(n=120 ; 60 \%)$ or along the $y$-axis anteriorly $(n=61 ; 31 \%)$ to obtain good coregistration. Adjustments in 2 or 3 planes had to be made in most cases $(n=176 ; 88 \%)$. Misregistration was not related to sex $(P=0.07)$, rest-versus-stress state $(P=0.58)$, or the camera used $(P=0.31)$ and did not correlate with patient age or weight. Analysis of reproducibility of the reregistration tool revealed a good intraobserver variability (weighted $\kappa$ of 0.66 for the experienced observer) and a fair interobserver variability (best weighted $\kappa$ of 0.39 for pairs of observers).

Figure 2 shows a representative example of SPECT/CT misalignment and its correction by reregistration.

\section{Regional Radiotracer Distribution}

The measured regional radiotracer uptake in percentage of maximal pixel intensity for 6 myocardial segments (derived from polar maps using the Cedars-Sinai QPS system) is shown in Tables 1 and 2. At univariate analysis, segmental regional radiotracer distribution differed between noncorrected and both types of attenuation-corrected images in all 6 evaluated regions $(P<0.0001$ for all $)$. Furthermore, there was a significant difference in radiotracer distribution between attenuation-corrected images before and after reregistration in the anterior $(P=0.044)$, septal $(P=0.004)$, inferior $(P=0.003)$, and anteroapical segments $(P=0.006)$.

\section{Multivariate Analysis of the Effect of Misregistration on Regional Radiotracer Distribution}

According to this analysis, misregistration was a significant independent contributor to changes in radiotracer uptake between noncorrected and attenuation-corrected images before realignment in the anterior $(P=0.038)$, septal $(P=$ $0.011)$, and inferior segments $(P=0.006)$. Misregistration was also an independent contributor to changes in regional radiotracer distribution in attenuation-corrected images before and after reregistration in the anterior $(P=0.0006)$, septal $(P=0.0003)$, inferior $(P=0.0006)$, and inferoapical segments $(P=0.046)$.

\section{Subgroup Analysis}

To create a subgroup of studies without obvious regional abnormalities, we excluded 61 datasets (23 datasets with truncation of the CT data and 38 datasets that were clinically interpreted as ischemia or infarction during a consensus reading). In the remaining 138 datasets, the results were unchanged compared with the entire group, and misalignment remained an independent determinant of regional tracer distribution on attenuation-corrected images.

In this subset of expected homogeneous tracer distribution, we also calculated a coefficient of variation for radiotracer uptake in the entire myocardium (sum of 6 segments) as a marker of overall radiotracer homogeneity. Notably, the coefficient of variation was significantly higher in noncorrected (coefficient of variation, $15.9 \% \pm 4.9 \%$ ) than attenuation-corrected images, both before (coefficient of variation, $9.3 \% \pm 3.8 \%$ ) and after (coefficient of variation, $10.2 \% \pm 4.0 \%)$ reregistration $(P<0.0001)$, whereas there was no significant difference between both attenuationcorrected datasets.

\section{DISCUSSION}

Our quantitative analysis shows that misregistration of CT and SPECT frequently occurs in myocardial perfusion SPECT/CT. Approximately two thirds of studies in our analysis showed a maximal misalignment of more than one SPECT pixel. Misregistration affects regional radiotracer distribution on attenuation-corrected SPECT images, and this effect is independent of other factors known to influence attenuation and image quality such as body weight, sex, or radiotracer photon energy. Finally, software-based realignment of CT and SPECT is a useful tool for correction, as suggested by its independent inverse effect on radiotracer distribution in the segments most influenced by misalignment without correction. Reproducibility of softwarebased interactive realignment, however, may vary with reader experience, suggesting that further, observer-independent strategies to correct or prevent misalignment are needed. These results have practical implications for the clinical use of CT-based attenuation correction in SPECT/CT. 
A

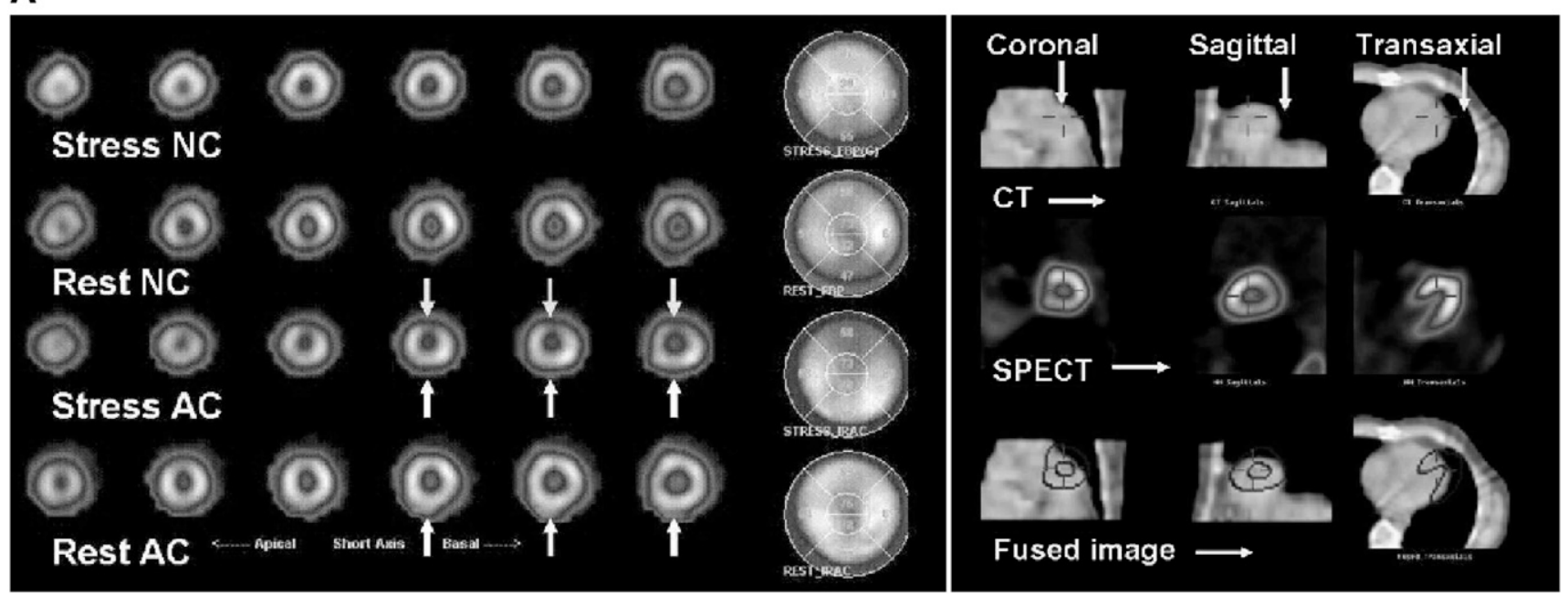

B

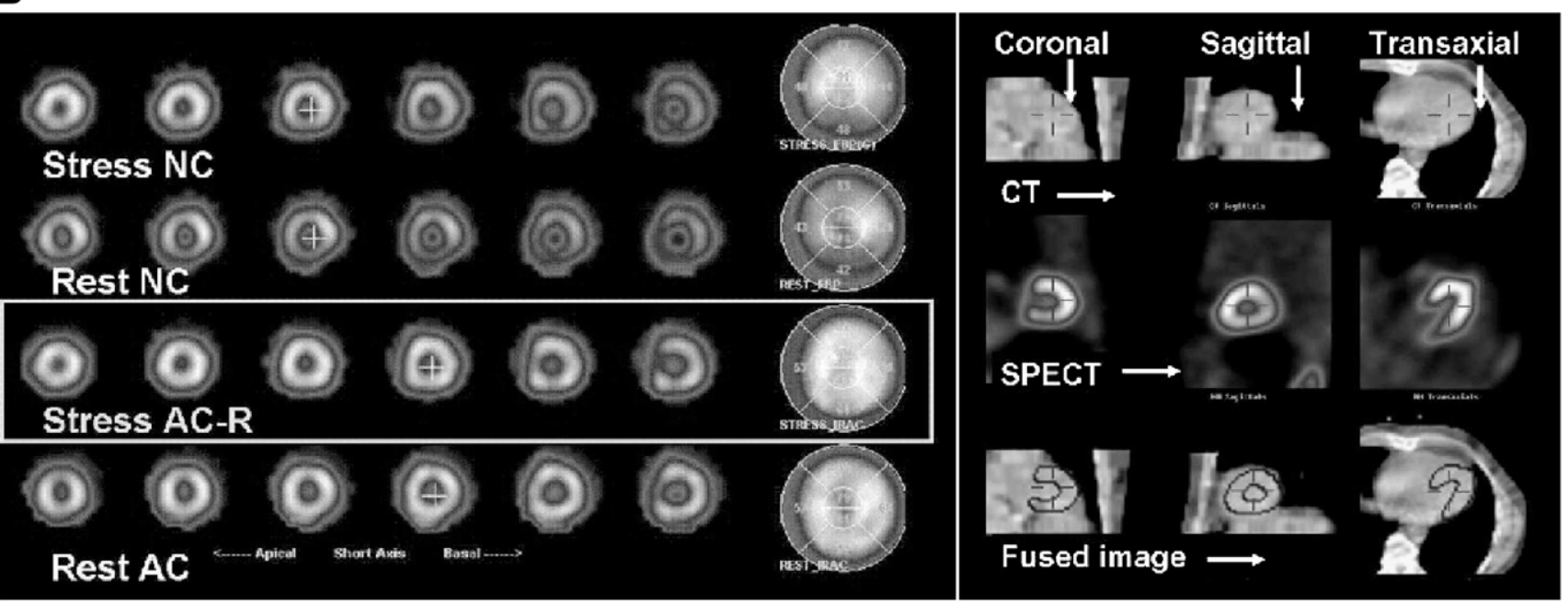

FIGURE 2. Representative case of SPECT/CT misalignment before (A) and after (B) reregistration. Stress and rest short-axis slices and polar maps are displayed on left. (A) Noncorrected images (NC) show decreased radiotracer uptake in inferior wall, compared with attenuation-corrected images (AC). Inferior wall uptake is improved, suggesting attenuation artifact on noncorrected images. Anterior wall, however, shows reduced uptake on attenuation-corrected vs. noncorrected images. Significant misalignment of SPECT and CT is shown in fusion images on right. (B) After best possible realignment (right), tracer distribution in attenuation-corrected images at stress and rest is homogeneous (left). AC- $\mathrm{R}=$ attenuation-corrected images after reregistration.

Attenuation correction increases the diagnostic accuracy of myocardial perfusion SPECT and is thus recommended for clinical use (1). It has, however, also added methodologic complexity and new aspects of quality control. SPECT/CT systems are increasingly successful and more widely applied. With these scanners, the use of CT for attenuation correction is attractive because a high-quality attenuation map results. The main concern in quality control of this attenuation correction algorithm is alignment of the SPECT and CT scans because the scans are acquired sequentially, leaving room for patient motion, respiratory motion, or mechanical misalignment.

A recent study by Masood et al. demonstrated that CTbased attenuation correction of SPECT using a dual-head SPECT/CT device improved the overall diagnostic accuracy of myocardial perfusion imaging and is therefore well suited for routine clinical use (7). Patients with misregistration between transmission and emission scans were excluded from this analysis, and specifics about the degree of misregistration that led to exclusion were not provided. Although the attenuation correction algorithm thus improved accuracy in cases of good alignment, no conclusions can be made about the effect of misalignment in that clinical study.

Several phantom and human studies, using different setups for attenuation correction, have shown that misregistration between emission and transmission data can produce significant errors in the activity distribution of the corrected images (3-6). Studies using CT-based attenuation correction in simulated data from humans and phantoms found that misregistration of $2-3 \mathrm{~cm}$ leads to a $20 \%-35 \%$ change in 
TABLE 1

Regional Radiotracer Uptake for Noncorrected and Attenuation-Corrected Myocardial Perfusion SPECT Images

\begin{tabular}{|c|c|c|c|}
\hline \multirow[b]{3}{*}{ Area } & \multirow[b]{2}{*}{ Mean $\pm S D$} & \multicolumn{2}{|c|}{$\mathrm{NC}$ to $\mathrm{AC}$} \\
\hline & & \multirow[b]{2}{*}{ Difference } & \multirow{2}{*}{$\begin{array}{c}95 \% \\
\text { confidence } \\
\text { interval }\end{array}$} \\
\hline & $\mathrm{AC}$ & & \\
\hline Anterior & $61.5 \pm 7.164 .1 \pm 6.4$ & 2.57 & $1.86,3.28$ \\
\hline Lateral & $64.6 \pm 6.268 .4 \pm 5.3$ & 3.82 & $3.06,4.59$ \\
\hline Septal & $58.9 \pm 7.662 .5 \pm 8.1$ & 3.59 & $2.72,4.47$ \\
\hline Inferior & $55.0 \pm 9.064 .5 \pm 7.8$ & 9.45 & $8.53,10.4$ \\
\hline Anteroapical & $77.0 \pm 6.971 .7 \pm 7.0$ & -5.33 & $6.17,-4.49$ \\
\hline Inferoapical & $77.3 \pm 7.473 .9 \pm 6.9$ & -3.44 & 4.40. -2.49 \\
\hline
\end{tabular}

$\mathrm{AC}=$ attenuation-corrected; $\mathrm{NC}=$ noncorrected.

Data are percentage maximal pixel intensity for each of 6 evaluated segments of left ventricle, with NC-to-AC differences and $95 \%$ confidence intervals.

radiotracer distribution (5) and that a 7-mm change can lead up to a $15 \%$ change in regional tracer uptake (6). A more recent study, by Fricke et al. (3), suggested that a misalignment of 1 pixel can lead to artifacts in the anterior, apical, and septal segments. That study specifically selected patients with normal noncorrected images who appeared to have artificial defects after attenuation correction. Another recent study, by Tonge et al. (2), found that attenuation correction in SPECT/CT consistently improved radiotracer distribution in the inferior wall, but the investigators also concluded that minor degrees of misregistration between emission and transmission images increased the likelihood of creating a defect. The report, however, was based on qualitative visual assessment and did not use quantitative techniques.

Effects are most likely less clear in heterogeneous clinical populations than in simulated data or phantom data because

TABLE 2

Regional Radiotracer Uptake for Attenuation-Corrected Myocardial Perfusion SPECT Images Before and After Reregistration

\begin{tabular}{|c|c|c|c|}
\hline \multirow[b]{3}{*}{ Area } & \multirow[b]{2}{*}{ Mean $\pm S D$} & \multicolumn{2}{|c|}{$A C$ to $R R$} \\
\hline & & \multirow[b]{2}{*}{ Difference } & \multirow{2}{*}{$\begin{array}{c}95 \% \\
\text { confidence } \\
\text { interval }\end{array}$} \\
\hline & $\mathrm{RR}$ & & \\
\hline Anterior & $64.1 \pm 6.463 .3 \pm 6.8$ & -0.81 & $1.13,-0.43$ \\
\hline Lateral & $68.4 \pm 5.367 .7 \pm 5.7$ & -0.69 & $1.03,-0.35$ \\
\hline Septal & $62.5 \pm 8.161 .1 \pm 8.4$ & -1.40 & $1.76,-1.03$ \\
\hline Inferior & $64.5 \pm 7.862 .7 \pm 8.0$ & -1.71 & $2.10,-1.32$ \\
\hline Anteroapical & $71.7 \pm 7.073 .0 \pm 7.0$ & 1.33 & $0.90,1.75$ \\
\hline Inferoapical & $73.9 \pm 6.974 .4 \pm 6.5$ & 0.52 & $0.07,0.97$ \\
\hline
\end{tabular}

$\mathrm{AC}=$ attenuation-corrected before reregistration; $\mathrm{RR}=$ attenuationcorrected after reregistration.

Data are percentage maximal pixel intensity for each of 6 evaluated segments of left ventricle, with AC-to-RR differences and $95 \%$ confidence intervals. misregistration in humans is complex (and often happens in more than one dimension of space). Our study, using unselected clinical cases, described quantitative changes in radiotracer distribution in various segments of the myocardium, with an increase especially in the inferior wall, but also in the anterior, septal, and lateral walls, accompanied by a decrease in radiotracer distribution in the apical segments. This finding seems to correspond well to previously described more prominent apical thinning with attenuation correction (89). We also showed that misregistration, when measured quantitatively, affects regional radiotracer distribution in the anterior, septal, and inferior walls and that reregistration of misalignment significantly affected regional radiotracer distribution in the segments shown to be influenced by misregistration. Our results emphasize the importance of attenuation correction quality control and of a further clinical evaluation of reregistration tools (10). Reregistration with the software tool used in our study was fairly complex, requiring several steps. This complexity led to subjectivity as reflected in the good intraobserver, but only fair interobserver, variability. Therefore, it is important to investigate other methods of realignment (e.g., automated approaches) and measures to prevent misregistration (optimized protocols to increase patient comfort, adapted breathing protocols, ultra slow acquisition or respiration-averaged CT, respiratory gating) in the future (11).

\section{CONCLUSION}

Particularly with the increasing use of SPECT/CT systems and the technical advances in these imaging systems, evaluation for transmission-emission misregistration and its effects is important for appropriate attenuation correction. Reregistration strategies will be helpful in avoiding artifacts related to misregistration. The impact of misregistration (and its correction) on the diagnostic and prognostic accuracy of myocardial perfusion studies needs to be determined in future investigations, and implications for SPECT/CT and PET/CT systems with faster multislice CT components need to be determined.

\section{ACKNOWLEDGMENTS}

Preliminary results from this study were presented as an abstract at the ASNC 2006 annual meeting and at the AHA 2006 scientific sessions.

\section{REFERENCES}

1. Heller GV, Links J, Bateman TM, et al. American Society of Nuclear Cardiology and Society of Nuclear Medicine joint position statement: attenuation correction of myocardial perfusion SPECT scintigraphy. J Nucl Cardiol. 2004;11:229-230.

2. Tonge CM, Manoharan M, Lawson RS, Shields RA, Prescott MC. Attenuation correction of myocardial SPECT studies using low resolution computed tomography images. Nucl Med Commun. 2005;26:231-237.

3. Fricke H, Fricke E, Weise R, Kammeier A, Lindner O, Burchert W. A method to remove artifacts in attenuation-corrected myocardial perfusion SPECT introduced by misalignment between emission scan and CT-derived attenuation maps. J Nucl Med. 2004;45:1619-1625. 
4. Matsunari I, Boning G, Ziegler SI, et al. Effects of misalignment between transmission and emission scans on attenuation-corrected cardiac SPECT. J Nucl Med. 1998;39:411-416.

5. Stone CD, McCormick JW, Gilland DR, Greer KL, Coleman RE, Jaszczak RJ. Effect of registration errors between transmission and emission scans on a SPECT system using sequential scanning. J Nucl Med. 1998;39:365-373.

6. Takahashi Y, Murase K, Higashino H, Mochizuki T, Motomura N. Attenuation correction of myocardial SPECT images with x-ray CT: effects of registration errors between X-ray CT and SPECT. Ann Nucl Med. 2002;16:431-435.

7. Masood Y, Liu YH, Depuey G, et al. Clinical validation of SPECT attenuation correction using X-ray computed tomography-derived attenuation maps: multicenter clinical trial with angiographic correlation. J Nucl Cardiol. 2005;12:676-686.
8. Links JM, Becker LC, Anstett F. Clinical significance of apical thinning after attenuation correction. J Nucl Cardiol. 2004;11:26-31.

9. O'Connor MK, Kemp B, Anstett F, et al. A multicenter evaluation of commercial attenuation compensation techniques in cardiac SPECT using phantom models. J Nucl Cardiol. 2002;9:361-376.

10. Chen J, Caputlu-Wilson SF, Shi H, Galt JR, Faber TL, Garcia EV. Automated quality control of emission-transmission misalignment for attenuation correction in myocardial perfusion imaging with SPECT-CT systems. J Nucl Cardiol. 2006; 13:43-49.

11. Martinez-Möller A, Souvatzoglou M, Navab N, Schwaiger M, Nekolla SG. Artifacts from misaligned CT in cardiac perfusion PET/CT studies: frequency, effects, and potential solutions. J Nucl Med. 2007;48:188-193.

\section{Erratum}

In the article "Cardiac Image Fusion from Stand-Alone SPECT and CT: Clinical Experience," by Gaemperli et al. ( $\mathrm{J} \mathrm{Nucl} \mathrm{Med.} \mathrm{2007;48:696-703),} \mathrm{Table} 2$ contains an error for patient 33. The correct data for that patient are given below. In addition, the data for patient 23 in Table 2 contained a typographic error. "qMid" should have been "Mid." We regret the errors.

TABLE 2

Results of Nonfused and Fused SPECT/CT Analysis

\begin{tabular}{|c|c|c|c|c|c|c|c|}
\hline \multirow{2}{*}{$\begin{array}{l}\text { Patient } \\
\text { no. }\end{array}$} & \multirow[b]{2}{*}{ Sex } & \multirow{2}{*}{$\begin{array}{l}\text { Age } \\
\text { (y) }\end{array}$} & \multirow{2}{*}{$\begin{array}{c}\text { CTA results: } \\
\text { coronary segment } \\
\text { with stenosis }>50 \%\end{array}$} & \multicolumn{2}{|c|}{$\begin{array}{c}\text { MPI results: perfusion } \\
\text { defect }\end{array}$} & \multicolumn{2}{|c|}{ Culprit vessel: analysis } \\
\hline & & & & Reversible & Fixed perfusion & Separate nonfused & Fused \\
\hline 33 & $\mathrm{~F}$ & 75 & $1,3,6,7$ & Apical & Inferior & $\begin{array}{l}\text { Prox/mid LAD, } \\
\text { prox/mid RCA* }\end{array}$ & $\begin{array}{l}\text { Prox/mid LAD, } \\
\text { prox/mid RCA* }\end{array}$ \\
\hline
\end{tabular}

